\title{
Method to Control Multiple Segmented LLSM without Position Encoder
}

\author{
Andreas R. Weber and Gerald Steiner \\ Institute of Electrical Measurement and Measurement Signal Processing, Graz University of Technology, Austria, \\ e-mail: andreas.r.weber@sbg.at,gerald.steiner@ieee.org
}

\begin{abstract}
In recent years long stator linear synchronous motors (LLSM) started to replace the typical rotating to linear converters like belts, chains, screw systems, pulleys, disks and so on. With LLSM a lot of drawbacks in the machine concepts and design can be prevented. For long tracks, reducing reactive power and individual control of numerous moveable units, the track is segmented in several fed stator units. Because of the LLSM construction an operation with a physical position encoder is not desirable. For that reason position observers should be used instead. In this paper a method to control the multiple segmented LLSM without position encoder is proposed. The special behaviour during entrance and exit of one moveable unit in an active stator segment is described and the control concept via a master slave relationship is proposed. Experimental results are presented to demonstrate the performance and feasibility of the proposed method.
\end{abstract}

Keywords - long stator linear motor, short block motor, flux observer, encoderless, sensorless, multiple segmented

\section{INTRODUCTION}

In industrial applications most linear movements are solved using rotating machines in combination with rotating to linear converters like belts, chains, screw systems, pulleys, disks and similar. Generally these applications have not high requirements on position accuracy, speed variations and high dynamics. In recent years a trend from the classical solution with rotating motors with rotating to linear converters to direct drives systems with linear motors and preferably with LLSM emerged. The advantages of using linear direct drives in the case of transport of humans, goods or workpieces and in industrial manufacturing processes are diverse. Linear transmission units like belts, chains and so on corrupt an individual control of the moveable unit. There are also limitations in the range of use regarding acceleration and speed. Most linear transmission units are optimized for certain speed regions. That means they operate sufficiently only in low speed or high speed regions and with drawbacks in the opposite region. Furthermore it is not guaranteed that bidirectional movement is feasible.

By using LLSM the moveable units can be controlled individually. Thereby it is possible to discharge single units, for example a defect good or workpiece, from the product stream. The arisen space in processing can be easily filled with following moveable units. Moreover, it is possible to separate the moveable units and adapt the movers to diverse takeover stations with different clock cycles. Additional advantages are given in analysis and monitoring of the moveable units. Traced currents can be evaluated to indicate impending losses of a moveable unit. The recorded data for all moveable units can be used to improve the quality, energy utilization and especially to improve the flow rate of the whole machine. In short:

- improved utilization of the speed behaviour in the whole speed range,

- improved dynamic behaviour,

- individual control of the moveable units,

- improved energy utilization (less losses),

- reduced maintenance costs based on reduced mechanical wear parts,

- $\quad$ easy replacement and infiltration of the moveable units,

- monitoring, error detection,

- optimization of the product flow,

- increased flow rate,

- $\quad$ easy adapting of cyclic time adjustments (e.g. changing of transfer station).

On the other hand the number of the switching elements increases sharply. In simple terms the mechanical units are shifted into electrical units. Depending on the application, up to hundreds of moveable units can be deployed on the diverse track configurations. Assuming that the distance between the moveable units should be as small as possible and the requirement of the moveable units individual control, the number of independent stator segments with connected power electronics will be in the range of the moveable units number. This leads not only to increased cost but also to more effort in programmable design of the whole process. Also the requirements on a fast network communication increase. By using individual control of the moveable units it is essential to use modern and in field proofed control strategies, like the field oriented control [1], [2]. A typical controller structure for the current controller loop is shown in Fig. 1. Measured currents are transformed via the Clark and Park transformations into a rotor fixed coordinate system as actual values for the current controller. The current vector in the rotating field oriented coordinate system is given by a field (d-direction) and force (q-direction) components. The output of the current controller is a voltage also in dqcoordinates. This voltage vector is transformed via the Park and Clark transformations into a stator fixed coordinate system $(\alpha, \beta)$ and finally into a three-phase system $(\mathrm{u}, \mathrm{v}, \mathrm{w})$. The three-phase voltages are converted 
into switching times $\left(\mathrm{t}_{\mathrm{su}}, \mathrm{t}_{\mathrm{sv}}, \mathrm{t}_{\mathrm{sw}}\right)$ of the switching elements by using the measured DC-bus voltage. With the calculated times the switching elements connect either $\mathrm{U}_{\mathrm{dc}+}$ or $\mathrm{U}_{\mathrm{dc}-}$ with the motor windings.

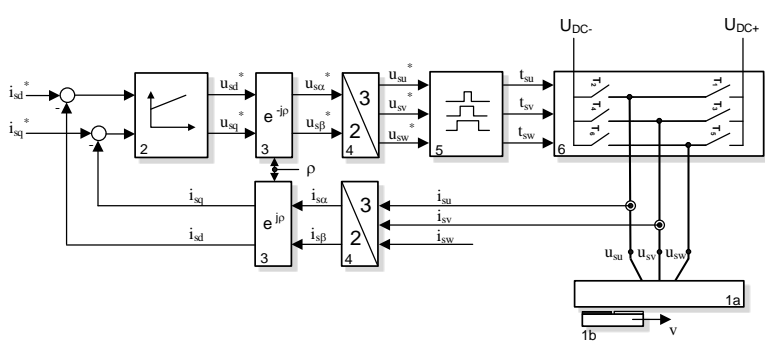

Fig. 1. Field oriented current control loop with a long stator linear motor segment. $1 \mathrm{a}$ - stator unit, $1 \mathrm{~b}$ - mover, 2 - current controller, 3 - Park transformation, 4 - Clarke transformation, 5 - time unit, 6 - switching elements.

The described field oriented control structure assumes exact information about the flux position. Typically a shaft encoder is used to provide the servo drive with this necessary position information. For lower investment costs, lower maintenance costs and increased reliability, the shaft encoder, necessary cables and evaluation unit are saved and replaced by an observer. For rotating motors, several methods for encoderless control are described in common literature [3], [4]. Those methods can be classified into two main groups. The first group is based on the fundamental model of the motor and uses the electromagnetic force as response of a moving system. The induced voltage observer or flux observer are used to obtain the rotor position. The induced voltage increases with increased speed and disappears at standstill. For that reason methods based on the fundamental model of the motor are unsuited for the position detection at standstill and near standstill. The second group uses effects like saturations, eccentricities and asymmetries in the motor structure to detect the position of the rotor. This methods work well at standstill and round standstill. Publications in reference to encoderless methods for linear drives are mainly focused on the short stator linear motor.

Various representatives use the model adaptive system estimator [5], kalman filter [6], HF signal injection method [7] or non-linear observer with an $\mathrm{H}$-inf estimator [8]. Very few publications can be found for the encoderless control of LLSM. Ref. [9] uses a direct torque method, [10] an observer in dq-reference frame, [11] an emf-observer and [12] an HF signal injection method, [11] and [12] have also a focus on the control of the moving element related to the transfer between the segments. The represented method is based on the central controller strategy. Both included segments are controlled by one speed controller. The output of the controller, in this case the stator current in quadrature direction, is connected to both inverters. The information of the commutation angle is estimated by two position observers based on the induced EMF voltage. Probably the biggest drawback is that all systemically important signals has to be send to the central controller and the set values back to the inverter units. This leads to huge requirements on the cyclic time and network communication, specifically by using a lot of segments.
In this paper a method to control the LLSM with multiple segments without position encoder is presented. A current controller is proposed which considers the characteristic of the flux during a transfer from the moveable unit through a segment. The used position observer bases on an expanded flux observer which also uses the information of the flux characteristic. The expanded observer is provided by a voltage observer to avoid voltage measurement of the phases. And finally a technique is presented to control the moveable unit from one segment to another. A requirement on the method is to use only standard inverter units, with standard network and without additional measurement equipment. The paper is organized as follows. In Section II the motor model from the fundamental voltage equation to the force equation is described. Also the inner loop of the controller cascade, the current controller loop, is explained. In Section III the proposed encoderless control method is presented and the consideration regarding the transfer between the stator segments are shown. In Section IV the experimental results are presented and Section V concludes the article.

\section{ANALYSIS}

The behaviour of the long stator linear synchronous motors is quite similar to rotating synchronous motors. The big difference is that the moveable unit is completely or partly overlapped with the active segment. That means that the flux depends on the position of the moveable unit. Fig. 2 describes this behaviour.

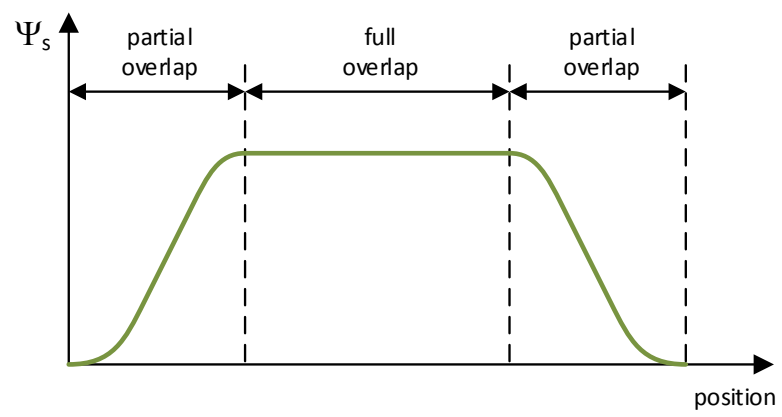

Fig. 2. Flux (absolute value) as function of the position of the moveable unit.

\section{A. Voltage Equation}

With the following simplifications:

- $\quad$ stator segments are structured in a three phase symmetric winding and can be transformed into an equivalent rotating two phase winding,

- current-fed windings produce a sinusoidal flux distribution along the air gap,

- the flux in the air gap is produced by the stator currents and permanent magnets of the moveable unit,

- the neutral (start) point of the windings is isolated,

- capacitive coupling is neglected,

- effects based on saturation are neglected, 
- additional force harmonics based on asymmetries, cogging and the like are neglected,

- the windings are fed by a symmetrical three phase system without a zero point component,

the voltage equation of long stator linear synchronous motors in a three phase coordinate system is

$$
u_{s 3}=R_{s 3} i_{s 3}+\frac{d \psi_{s 3}}{d t}
$$

with

$$
\begin{gathered}
u_{s 3}=\left[\begin{array}{c}
u_{s u} \\
u_{s v} \\
u_{s w}
\end{array}\right], R_{s 3}=\left[\begin{array}{ccc}
R_{s u} & 0 & 0 \\
0 & R_{s v} & 0 \\
0 & 0 & R_{s w}
\end{array}\right], \\
i_{s 3}=\left[\begin{array}{c}
i_{s u} \\
i_{s v} \\
i_{s w}
\end{array}\right], \psi_{s 3}=\left[\begin{array}{c}
\psi_{s u} \\
\psi_{s v} \\
\psi_{s w}
\end{array}\right] .
\end{gathered}
$$

With the mentioned simplifications and $\mathrm{R}_{\mathrm{s}}=\mathrm{R}_{\mathrm{su}}=\mathrm{R}_{\mathrm{sv}}=\mathrm{R}_{\mathrm{sw}}$ the transformation in the stator fixed coordinate system $(\alpha \beta)$ gives

$$
u_{s \alpha \beta}=R_{s} i_{s \alpha \beta}+\frac{d \psi_{s \alpha \beta}}{d t}
$$

with

$$
u_{s \alpha \beta}=\left[\begin{array}{c}
u_{s \alpha} \\
u_{s \beta}
\end{array}\right], i_{s \alpha \beta}=\left[\begin{array}{c}
i_{s \alpha} \\
i_{s \beta}
\end{array}\right], \psi_{s \alpha \beta}=\left[\begin{array}{c}
\psi_{s \alpha} \\
\psi_{s \beta}
\end{array}\right] .
$$

The stator flux can be calculated from the contributions of the stator currents in the stator windings and the permanent magnets of moveable unit.

$$
\psi_{s \alpha \beta}=L_{s \alpha \beta} i_{s \alpha \beta}+\psi_{p \alpha \beta}
$$

with

$$
L_{s \alpha \beta}=\left[\begin{array}{cc}
L_{\alpha \alpha} & L_{\alpha \beta} \\
L_{\beta \alpha} & L_{\beta \beta}
\end{array}\right], \psi_{p \alpha \beta}=\left[\begin{array}{c}
\psi_{p \alpha} \\
\psi_{p \beta}
\end{array}\right] .
$$

The voltage equation (3) expanded with the equation of the stator flux (5) and relation of the position $x$, commutation angle $\rho$ and pole width $\tau_{\mathrm{p}}$

$$
\rho=\frac{\pi}{\tau_{p}} x
$$

leads to

$u_{s \alpha \beta}=\underbrace{R_{s} i_{s \alpha \beta}}_{I}+\underbrace{L_{s \alpha \beta} \frac{d i_{s \alpha \beta}}{d t}}_{I I}+\underbrace{\frac{\partial L_{s \alpha \beta}}{\partial x} \frac{d x}{d t} i_{s \alpha \beta}}_{I I I}+\underbrace{\frac{\partial \psi_{p \alpha \beta}}{\partial x} \frac{d x}{d t}}_{I V}$.

The first term I. is the ohmic voltage drop, II. the inductive voltage drop following the stator current changes, IV. the voltage drop based on the electromagnetic force (EMF) and III. a voltage drop based on the reluctance differences. The description of the motor model in the stator fixed coordinate system is used for the flux observer for detecting the position of the flux and thus of the moveable unit. From the control perspective a transformation into a field oriented coordinate system (dq) is useful.

$$
u_{s d q}=R_{s} i_{s d q}+\frac{d \psi_{s d q}}{d t}+j \frac{\pi}{\tau_{p}} \frac{d x}{d t} \psi_{s d q}
$$

$$
u_{s d q}=\left[\begin{array}{c}
u_{s d} \\
u_{s q}
\end{array}\right], i_{s d q}=\left[\begin{array}{c}
i_{s d} \\
i_{s q}
\end{array}\right], \psi_{s d q}=\left[\begin{array}{c}
\psi_{s d} \\
\psi_{s q}
\end{array}\right]
$$

The stator flux can be calculated again as a combination of the stator currents in the stator windings and the permanent magnets of the moveable unit

$$
\psi_{s d q}=L_{s d q} i_{s d q}+\psi_{p d q}
$$

with

$$
L_{s d q}=\left[\begin{array}{cc}
L_{s d} & 0 \\
0 & L_{s q}
\end{array}\right], \psi_{p d q}=\left[\begin{array}{c}
\psi_{p} \\
0
\end{array}\right] .
$$

Included in (9) and grouped

$$
\begin{array}{r}
u_{s d q}=R_{s} i_{s d q}+L_{s d q} \frac{d i_{s d q}}{d t}+\frac{\partial \psi_{p d q}}{\partial x} \frac{d x}{d t}+ \\
j \frac{\pi}{\tau_{p}}\left(L_{s d q} i_{s d q}+\psi_{p d q}\right) \frac{d x}{d t} .
\end{array}
$$

Under the assumption that the defined permanent magnet flux $\Psi_{\mathrm{p}}(13)$ is fixed with the d-direction of the coordinate system, the voltage equation in the field oriented coordinate system is given as follows

$$
\begin{aligned}
& u_{s d}=\underbrace{R_{s} i_{s d}}_{I}+\underbrace{L_{s d} \frac{d i_{s d}}{d t}}_{I I}+\underbrace{\frac{\partial \psi_{p}}{\partial x} \frac{d x}{d t}}_{V}-\underbrace{\frac{\pi}{\tau_{p}} L_{s q} i_{s q} \frac{d x}{d t}}_{I I I} \\
& u_{s q}=\underbrace{R_{s} i_{s q}}_{I}+\underbrace{L_{s q} \frac{d i_{s q}}{d t}}_{I I}+\underbrace{\frac{\pi}{\tau_{p}} \psi_{p} \frac{d x}{d t}}_{I V}+\underbrace{\frac{\pi}{\tau_{p}} L_{s d} i_{s d} \frac{d x}{d t}}_{I I I} .
\end{aligned}
$$

Term I. is again the ohmic voltage drop, II. the inductive voltage drop following the stator current changes, III. a voltage drop based on the coupling of the stator flux $\mathrm{L}_{\text {sdq }} \mathrm{i}_{\text {sdq }}$, IV. the voltage drop based on the electromagnetic force (EMF) only in the quadrature direction and V. a voltage drop which is based on the changes of the flux in dependence of the position. This term occurs mainly during entrance and exit of the moveable unit in the stator segment and only in the direct component of the stator voltage $\mathrm{u}_{\mathrm{sdq}}$.

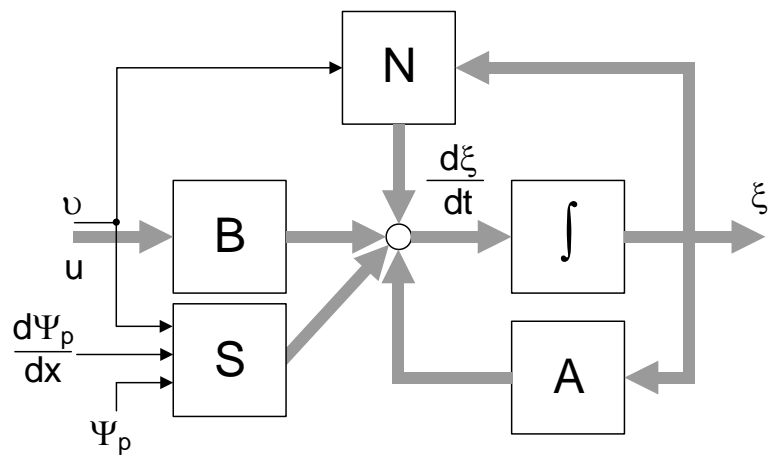

Fig. 3. Continuous state space model of a long stator linear synchronous motor in filed oriented coordinates.

\section{B. Thrust Force Equation}

The thrust force can be calculated via the electric power

$$
P_{e l}=i_{s 3}^{T} u_{s 3}=\frac{3}{2} i_{s \alpha \beta}^{T} u_{s \alpha \beta}=\frac{3}{2} i_{s d q}^{T} u_{s d q} .
$$

with 
With the voltage equation (14), the power can be written as

$$
\begin{gathered}
P_{e l}=\underbrace{\frac{3}{2} i_{s d q}^{T} R_{s} i_{s d q}}_{I}+\underbrace{\frac{3}{2} i_{s d q}^{T} L_{s d q} \frac{d i_{s d q}}{d t}}_{I I}+ \\
+\underbrace{\frac{3}{2} i_{s d q}^{T}\left[\frac{\partial \psi_{p d q}}{\partial x}+j \frac{\pi}{\tau_{p}}\left(L_{s d q} i_{s d q}+\psi_{p d q}\right)\right] \frac{d x}{d t}}_{I I I} .
\end{gathered}
$$

Term I. are the ohmic power losses, II. the power to store the magnetic energy and III. the part for the mechanical power. Thus the thrust force $\mathrm{F}$ can be written as

$$
F=\frac{3}{2} i_{s d q}^{T}\left[\frac{\partial \psi_{p d q}}{\partial x}+j \frac{\pi}{\tau_{p}}\left(L_{s d q} i_{s d q}+\psi_{p d q}\right)\right] .
$$

Multiplied and grouped the thrust force $\mathrm{F}$ can be rewritten as follows

$F=\frac{3}{2}\left\{i_{s d} \frac{\partial \psi_{p}}{\partial x}+\frac{\pi}{\tau_{p}}\left[\psi_{p} i_{s q}+i_{s d} i_{s q}\left(L_{s d}-L_{s q}\right)\right]\right\}$.

The thrust force $F$ consists of a main force $F_{h} \propto \Psi_{p} i_{s q}$, a force based on the reluctance differences between direct and quadrature inductances $\left(\mathrm{L}_{\mathrm{sd}}-\mathrm{L}_{\mathrm{sq}}\right)$ and an additional force term which is generated by the first deviation of the permanent flux over the position multiplied by the current in the direct direction $i_{\text {sd }}$. When using the field oriented control the current vector should be controlled typically to be normal to the permanent flux. This means that the current in the direct direction should be controlled to zero, $\left(i_{\text {sd }}=0\right)$. This simplifies the thrust force equation to

$$
F=\frac{3}{2} \frac{\pi}{\tau_{p}} \psi_{p} i_{s q} .
$$

\section{State Space Model}

From the control point of view a transformation of (14) into the state space with state space vector $\xi=\left[\begin{array}{ll}i_{\mathrm{sd}} & \mathrm{i}_{\mathrm{sq}}\end{array}\right]^{\mathrm{T}}$ is useful. Thus

$$
\begin{aligned}
& \underbrace{\frac{d}{d t}\left[\begin{array}{c}
i_{s d} \\
i_{s q}
\end{array}\right]}_{\dot{\xi}}=\underbrace{\left[\begin{array}{cc}
-\frac{1}{T_{s d}} & 0 \\
0 & \frac{1}{T_{s q}}
\end{array}\right]}_{A} \underbrace{\left[\begin{array}{c}
i_{s d} \\
i_{s q}
\end{array}\right]}_{\xi}+ \\
& \underbrace{\left[\begin{array}{cc}
\frac{1}{L_{s d}} & 0 \\
0 & \frac{1}{L_{s q}}
\end{array}\right]}_{B} \underbrace{\left[\begin{array}{c}
u_{s d} \\
u_{s q}
\end{array}\right]}_{u}+ \\
& v \underbrace{\left[\begin{array}{cc}
0 & \frac{\pi}{\tau_{p}} \frac{L_{s q}}{L_{s d}} \\
-\frac{\pi}{\tau_{p}} \frac{L_{s d}}{L_{s q}} & 0
\end{array}\right]}_{N} \underbrace{\left[\begin{array}{c}
i_{s d} \\
i_{s q}
\end{array}\right]}_{\xi}+ \\
& v \underbrace{\left[\begin{array}{c}
-\frac{1}{L_{s d}} \frac{\partial \psi_{p}}{\partial x} \\
-\frac{1}{L_{s q}} \frac{\pi}{\tau_{p}} \psi_{p}
\end{array}\right]}_{S} .
\end{aligned}
$$

with

$$
T_{s d}=\frac{R_{s}}{L_{s d}}, T_{s q}=\frac{R_{s}}{L_{s q}}, v=\frac{d x}{d t}
$$

Fig. 3 describes this behavior in field oriented coordinates, with:

$$
\begin{array}{lll}
\text { A } & \ldots & \text { system matrix, } \\
B & \ldots & \text { input matrix, } \\
N & \ldots & \text { coupling matrix, and } \\
\text { S } & \ldots & \text { disturbance vector. }
\end{array}
$$

If the characteristic curve of the permanent flux (identification or simulation) and instantaneous speed $\mathrm{v}$ are well known the disturbance vector can be compensated via the feed forward control.

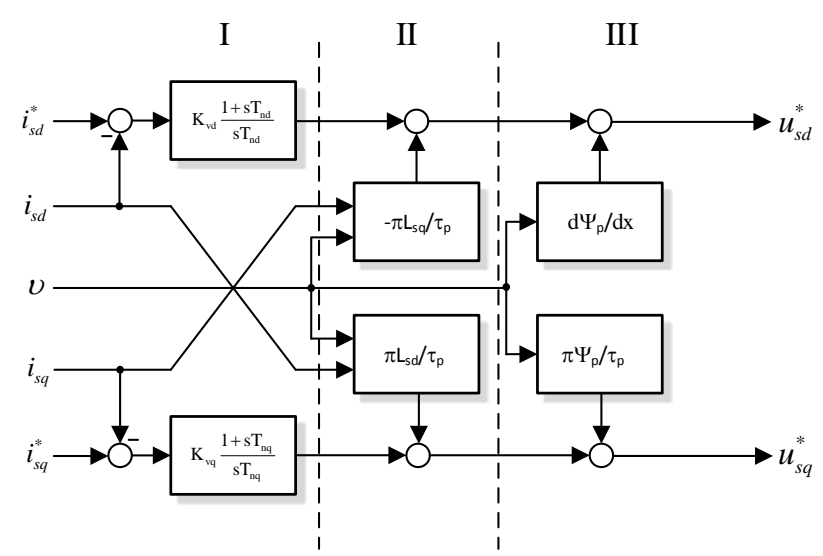

Fig. 4. Current controller with decoupling and feed forward.

With an additional decoupling network, which decouples the state variables, the state space model for the design of the current controller simplifies to

$$
\frac{d \xi}{d t}=A \xi+B u
$$

\section{Current Controller}

The current controller loop is the inner loop of the control structure and is described in Fig. 4. With the simplifications in C. the effort of the design of the current controller is reduced. The current controller consists of three parts. The first part should compensate the transient response which is described in the system matrix A. The response in each direction describes a PT1 behaviour with time constants $\mathrm{T}_{\mathrm{sd}}$ for the direct and $\mathrm{T}_{\mathrm{sq}}$ for the quadrature directions. In literature a lot of control strategies are developed to design this problem. In this work, two standard PI-controllers with the anti-windup procedure for each direction are selected. The second part deals with the decoupling of the state variables. Typically the current in the direct direction is controlled to zero, so a simplification in the decoupling network is given. Only one speed and $\mathrm{i}_{\mathrm{sq}}$-dependent voltage drop

$$
u_{s d, c}=v \frac{\pi}{\tau_{p}} L_{s q} i_{s q}
$$

remains in the direct direction. The third part is described through the disturbance vector $\mathrm{S}$ and is based on the speed dependent voltage drop through the flux $\Psi_{\mathrm{p}}$ and the deviation of the flux $\partial \Psi_{p} / \partial x$. Both voltage drops can be compensated by knowing the characteristic flux curve $\Psi_{\mathrm{p}}$ (x) and current speed v. Fig. 4 shows the current controller for both directions dq and the respective decoupling and feed forward strategies. 


\section{ENCODERLESS CONTROL}

The fundamental systems of linear motors are quite the same as typical rotating permanent magnet synchronous motors. So the methods for the position estimation of the rotating permanent magnet synchronous motors can be, in principle, as well used for linear motors. In the case of the long stator linear motors some additional differences and extensions have to be taken into account. The biggest difference is the overlapping of the moveable unit with the stator segment. This leads to a position dependent flux curve (Fig. 2), a lower EMF compared with rotating motors, and increased efforts for the control of the moveable unit during a transition.

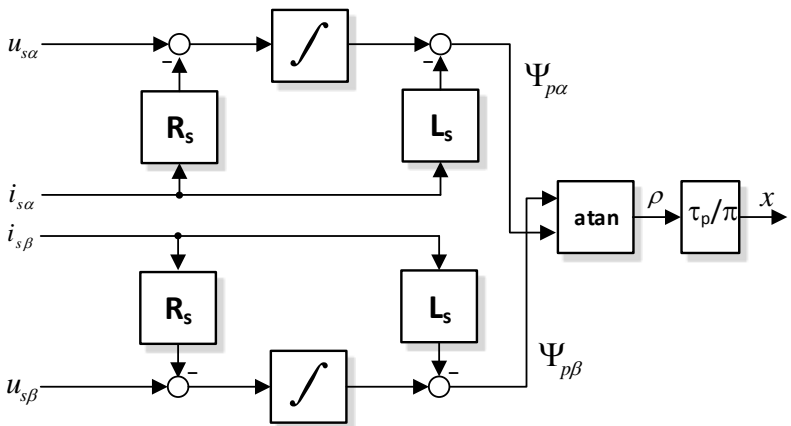

Fig. 5. Block diagram of the flux observer for the position estimation.

\section{A. Flux Observer}

The basis of the used observer for the position detection is a common flux observer in the stator fixed coordinate system $(\alpha \beta)$. Different methods of the flux observation for rotating motors have been published, and some examples are reported in [11], [13], [14]. For a robust and practicable use in industrial environment extensions regarding the non constant flux from entry to exit of the moveable unit are necessary. With (8) in the stator fixed coordinate system and $\mathrm{L}_{\mathrm{s} \alpha}=\mathrm{L}_{\mathrm{s} \beta}=\mathrm{L}_{\mathrm{s}}$ the voltage equations are given as

$$
\begin{aligned}
& u_{s \alpha}=R_{s} i_{s \alpha}+L_{s} \frac{d i_{s \alpha}}{d t}+\frac{\partial \psi_{p \alpha}}{\partial x} \frac{d x}{d t} \\
& u_{s \beta}=R_{s} i_{s \beta}+L_{s} \frac{d i_{s \beta}}{d t}+\frac{\partial \psi_{p \beta}}{\partial x} \frac{d x}{d t} .
\end{aligned}
$$

With the acknowledgement of the flux curve over the position, the flux in the stator fixed coordinate system can be written as

$$
\begin{aligned}
& \psi_{p \alpha}=\psi_{p} \cos \left(\frac{\pi}{\tau_{p}} x\right) \\
& \psi_{p \beta}=\psi_{p} \sin \left(\frac{\pi}{\tau_{p}} x\right)
\end{aligned}
$$

Using in equation (24), the rearranged and finally integrated over time the flux components are given as

$$
\begin{array}{r}
\underbrace{\int\left(u_{s \alpha}-R_{s} i_{s \alpha}\right) d t-L_{s} i_{s \alpha}}_{\psi_{p \alpha}}= \\
\int\left[\frac{\partial \psi_{p}}{\partial x} \cos \left(\frac{\pi}{\tau_{p}} x\right)-\frac{\pi}{\tau_{p}} \psi_{p} \sin \left(\frac{\pi}{\tau_{p}} x\right)\right] d x,
\end{array}
$$

$$
\begin{array}{r}
\underbrace{\int\left(u_{s \beta}-R_{s} i_{s \beta}\right) d t-L_{s} i_{s \beta}}_{\psi_{p \beta}}= \\
\int\left[\frac{\partial \psi_{p}}{\partial x} \sin \left(\frac{\pi}{\tau_{p}} x\right)+\frac{\pi}{\tau_{p}} \psi_{p} \cos \left(\frac{\pi}{\tau_{p}} x\right)\right] d x .
\end{array}
$$

The position $x$ of the moveable unit can be calculated via the arcus-tangens function.

$$
x=\frac{\tau_{p}}{\pi} \operatorname{atan}\left(\frac{\psi_{p \beta}}{\psi_{p \alpha}}\right)
$$

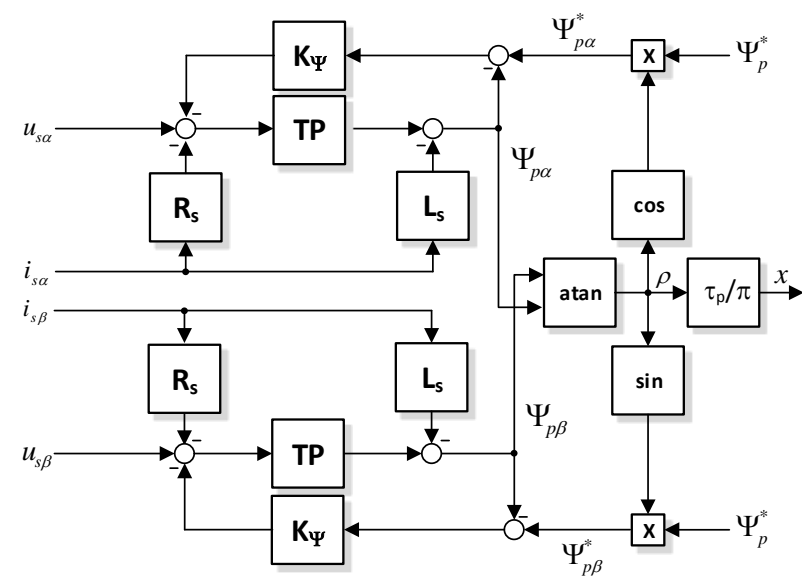

Fig. 6. Block diagram of the expanded flux observer for the position estimation with the low-pass filter instead of the integrator.

The described observer is shown in Fig. 5 and convinces with its simplicity. The biggest disadvantage is the open integration. Deviations in current, voltage and also in resistance lead to drifts in the stator flux $\Psi_{\mathrm{s}}$ and thus to errors in the position estimation. Ideally, the voltage is obtained via a direct measurement procedure. In most cases a direct measurement is not possible or unwanted because the measurement of the PWM

$$
\begin{aligned}
& \text { is complex and } \\
& \text { there are additional costs. }
\end{aligned}
$$

The set voltage of the current controller differs in essential ways from the real voltage. Errors are caused by the voltage drop of the connecting cable, nonlinear voltage drop of the switching elements as well as limitations in the control structure and quantization.

In [14] a voltage observer which considers those effects is presented and used in the application results to improve the quality of the position observer especially at low speed.

\section{B. Expanded Flux Observer}

The problem with the open integration of the observer structure is avoided with a low-pass filter and feedback term. This term is obtained via the difference of the calculated flux $\Psi_{\mathrm{p}}$ and an expected reference flux $\Psi_{\mathrm{p}}^{*}$ during a passage, see Fig. 2, multiplied by a gain factor $\mathrm{K}_{\Psi}$. Equations (27) and (28) extended with this feedback term leads to 


$$
\begin{aligned}
\psi_{p \alpha} & =\int\left(u_{s \alpha}-R_{s} i_{s \alpha}-K_{\psi} \Delta_{\psi p \alpha}\right) d t-L_{s} i_{s \alpha} \\
\psi_{p \beta} & =\int\left(u_{s \beta}-R_{s} i_{s \beta}-K_{\psi} \Delta_{\psi p \beta}\right) d t-L_{s} i_{s \beta}
\end{aligned}
$$

with

$$
\begin{aligned}
& \Delta_{\psi p \alpha}=\psi_{p \alpha}-\psi_{p}^{*} \cos \left(\frac{\pi}{\tau_{p}} x\right) \\
& \Delta_{\psi p \beta}=\psi_{p \beta}-\psi_{p}^{*} \sin \left(\frac{\pi}{\tau_{p}} x\right) .
\end{aligned}
$$

Fig. 6 shows a block diagram of this expanded flux observer for the position estimation. The estimated reference flux is mapped into the stator fixed components and compared with the correspondent components of the calculated flux.

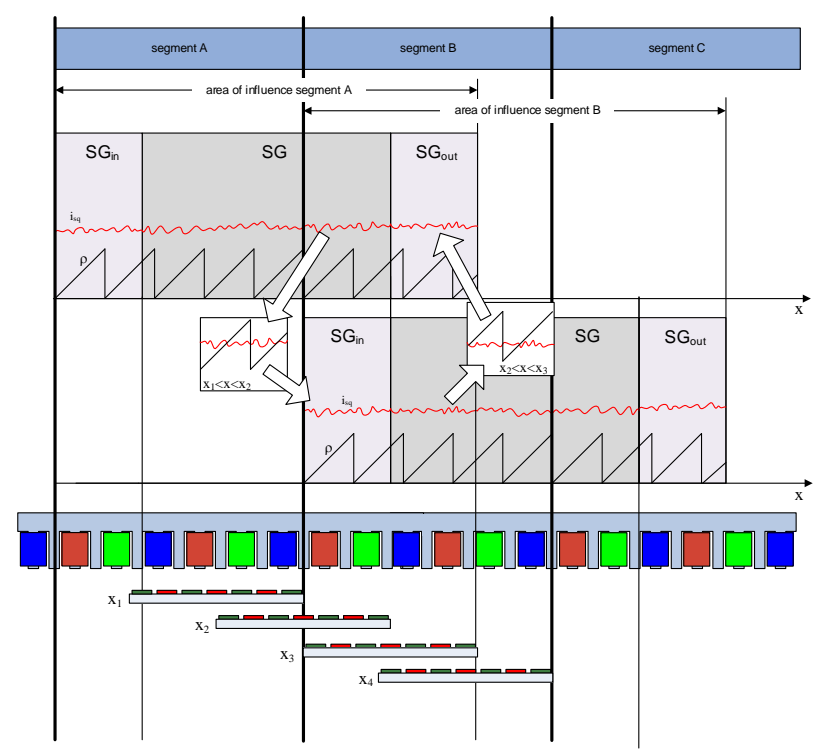

Fig. 7. Control strategy during a transfer from segment A to segment B.

The difference is added to the integrator or the used low-pass filter via a proportional element $\mathrm{K}_{\Psi}$. The wanted position $\mathrm{x}$ of the moveable unit can be calculated again via the arcus-tangens function.

\section{Multisegmented Control}

A particular consideration is the control of a moveable unit during a transfer from one segment to another one. The moveable unit is under influence of two separated inverter units. In this paper, a master slave connection between the involved segments is used. The segment with the higher overlap with the moveable unit, more precise with the higher overlap of the magnet area is the master and observed commutation angle $\rho$ and the output of the used speed controller $i_{\mathrm{sq}} *$ are sent to the slave segment to support the master segment. The connection between the two segments has to be fast. A constant high delay time $\Delta \mathrm{T}_{\text {com }}$ will lead to commutation errors and to disturbances in the quality of the moveable unit movement. For that reason a delay time compensation is suggested. A simple compensation assumes a constant speed $\mathrm{v}^{*}$ during the transfer and calculates the displacement of the commutation angle as

$$
\rho_{\text {corr }}=v^{*} \Delta T_{\text {com }} .
$$

The relationship between the two segments changes with the majority of the magnet overlap. The master segment changes into the slave segment and the slave segment changes to the master. With this, the direction of the sent values (current, compensated commutation angle) turns as well. This behaviour is shown in Fig. 7. With the position $\mathrm{x}_{1}$ the transfer starts. Until position $\mathrm{x}_{2}$, the segment $A$ sends the set current $i_{\mathrm{sq}}{ }^{*}$ and compensated commutation angle $\rho$ to the segment $\mathrm{B}$. The segment $\mathrm{B}$ receives the values and controls the currents in the coils with the sent set current and commutation angle. At the position $\mathrm{x}_{2}$ the relationship changes from master to slave and vice versa. Now the segment $B$ sends the set current and commutation angle and the segment $\mathrm{A}$ receives the data. With the position $\mathrm{x}_{3}$ the transfer is completed and the moveable unit is only in the influence of the segment $B$. At the position $\mathrm{x}_{4}$, the transfer between the segment $\mathrm{B}$ and segment $\mathrm{C}$ starts.

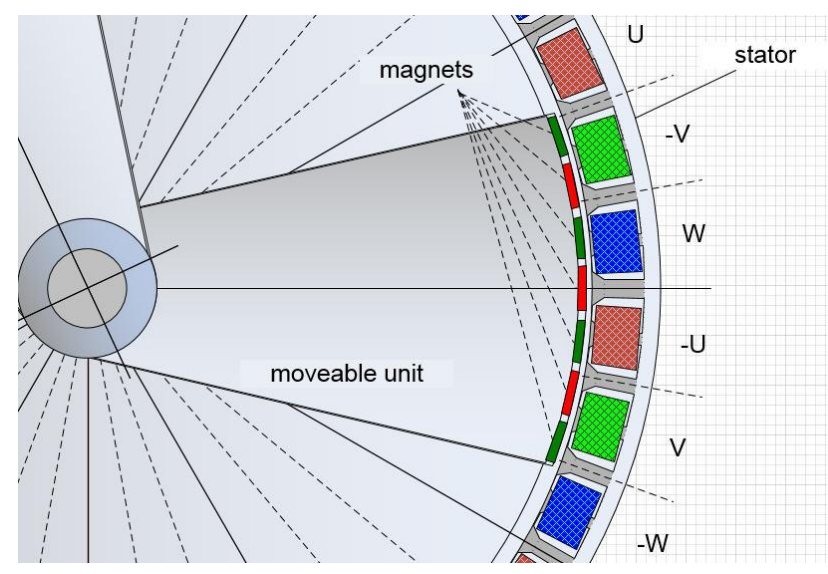

Fig. 8. Moveable unit and segment B of the used test motor.

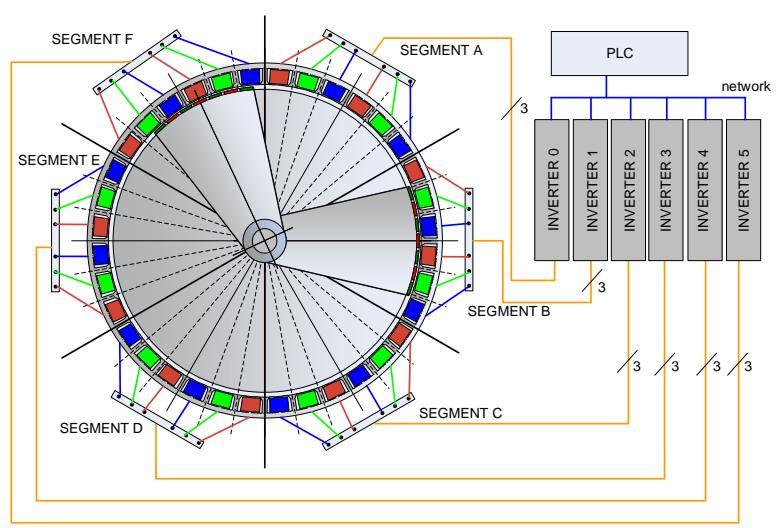

Fig. 9. Layout and connection of the used test motor with the fed inverter system and the programmable logic controller (PLC).

\section{APPLICATION RESULTS}

The used test motor is an adapted rotating torque motor. The stator geometry including winded and unwinded teeth and the yoke is identical. The start and end of the coil winding is connected with a clipboard for individual connecting. The stator is grouped into six identical 
segments with six coils. Two coils for each phase are connected in series and the ends of the three phases are combined into a star point. The rotor is split into two moveable units which are mounted separately. On each moveable unit 7 permanent magnets are affixed. For each moveable unit a rotating position encoder is mounted. The reference encoder is an optical encoder with a resolution of $2^{23}$ increments per turn. Fig. 8 shows one moveable unit with the permanent magnets, the coils for the segment B and a part of the stator. In Fig. 9, the layout and the connections are plotted. Motor parameters are described in the Appendix. In Fig. 10 the quality of the observed position in the section SG of the segment $\mathrm{A}$ is shown. The moveable unit passes the segment with a speed of $2.35 \mathrm{~m} / \mathrm{s}$. The observed position is compared with the connected encoder. The difference is shown in the lower figure. The difference of up to two mm corresponds to an error in the electrical commutation angle of 0.262 radiant or 15 degrees. The error (approx. 3.4\%) for the commutation is a range which can be neglected.
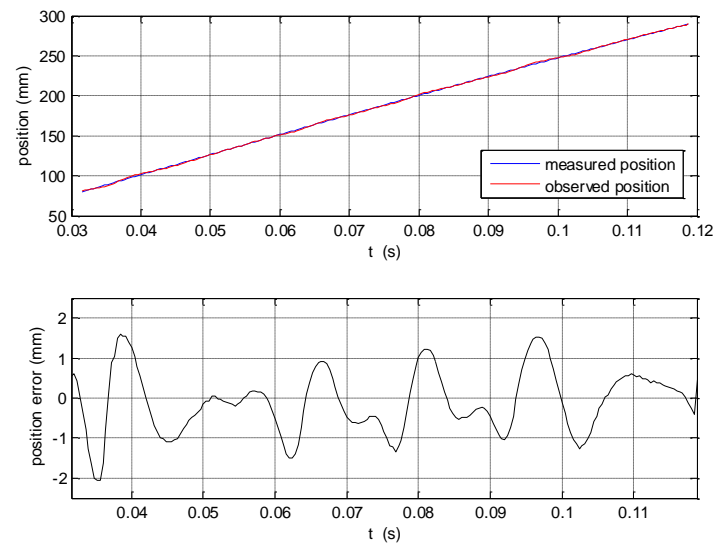

Fig. 10. Measured, observed position and the error of observation as soon as the moveable unit is majoritarian in the segment.

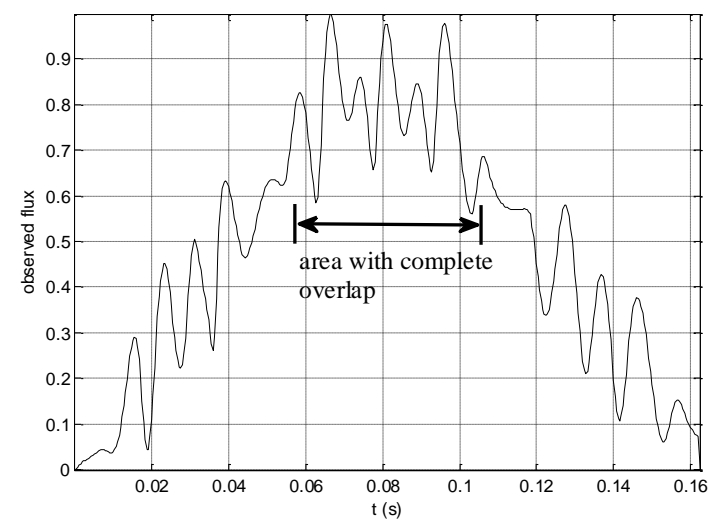

Fig. 11. Normalized observed flux (absolute value) during a full transition of a moveable unit through a segment.

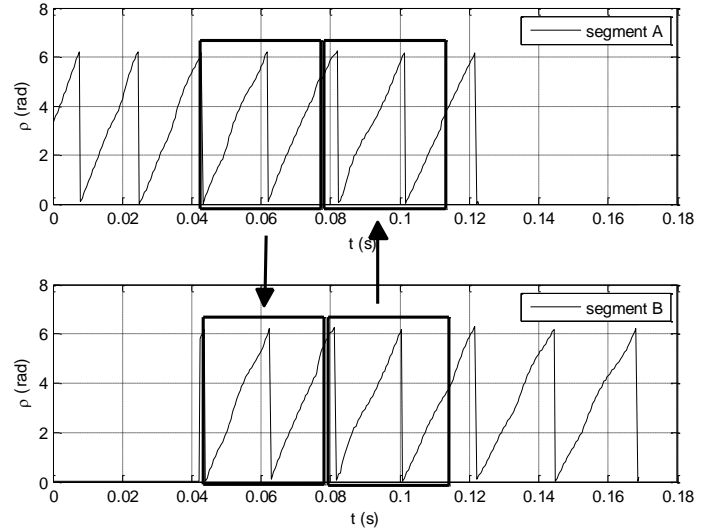

Fig. 12. Observed commutation angle $\rho$ during transfer from segment A to segment $\mathrm{B}$ with interchange from $\mathrm{A}$ to $\mathrm{B}$ and vice versa.

Fig. 11 shows the characteristics of the flux from entrance to exit of the moveable unit through a segment. In order to achieve the same quality of the observed position during a transfer from the segment $A$ to the segment $\mathrm{B}$, an interchange method of the quadrature current $i_{\mathrm{sq}}$ and the observed commutation angle $\rho$ from the segment A to the segment B and vice versa, as described in III.C., must be in this case performed. In Fig. 12 and Fig. 13 the commutation angle $\rho$ and quadrature current $\mathrm{i}_{\mathrm{sq}}$ during a transfer is shown.

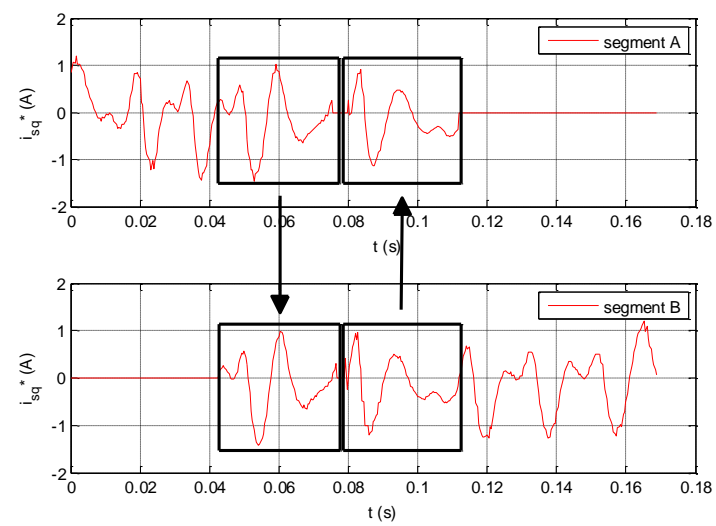

Fig. 13. Quadrature current $i_{\mathrm{sq}}$ during transfer from segment $A$ to segment B with interchange from A to B and vice versa.

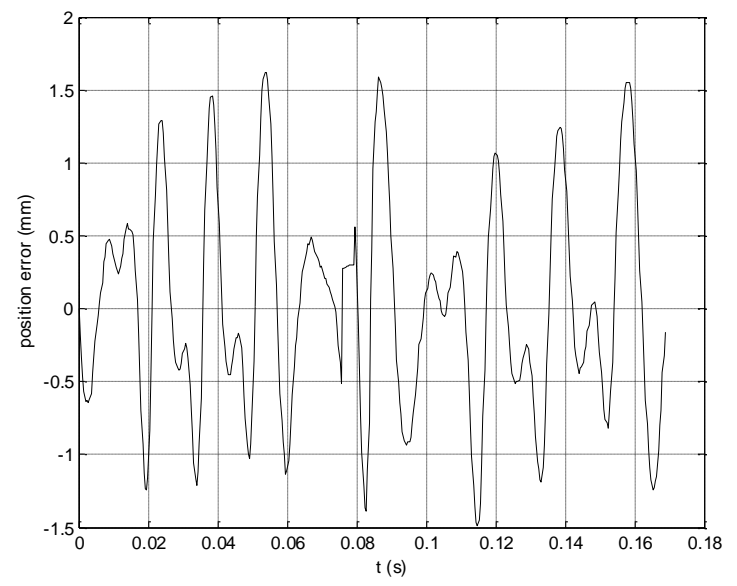

Fig. 14. Error of the observed position during transfer from segment A to segment B. 
The moveable unit starts in the segment A. After a time of approximately $0.042 \mathrm{~s}$ the moveable unit starts to enter in the segment B. From this time to approximately $0.078 \mathrm{~s}$ the segment A sends the information of the current and commutation angle to the segment B to get a full coupling with the flux. After this time the direction of the sent information changes. Now the moveable unit majority is in the segment B and sends the current and the commutation angle to the segment A. Between the change of the direction of the information a small gap in current appears and thus the thrust force occurs. This happens on the basis of an inadequacy in the implementation of the interchange. Because of the small time without thrust force the impact on the movement can be neglected. Fig. 14 shows the difference between the observed position compared with the connected encoder during a transfer from the segment $\mathrm{A}$ to the segment B. By using the presented interchange strategy the error during a transfer from segment to segment is in the same region as the error during a full overlap in one segment. In Fig. 15, the resulting flux during a full transition of a moveable unit through a segment is shown.

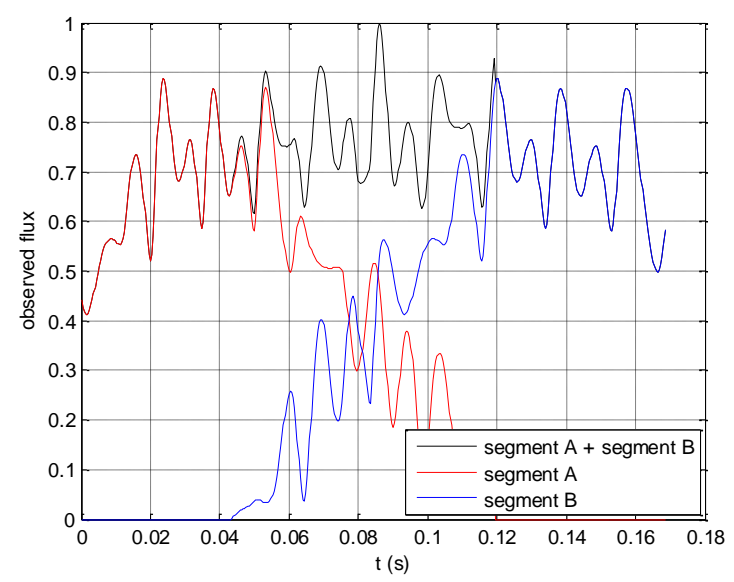

Fig. 15. Normalized observed flux (absolute value) from segment $A$ and segment $\mathrm{B}$ and the resulting flux (segment A plus segment B) during transfer from segment A to segment B.

\section{CONCLUSION}

This paper presents an accurate method to control multi segmented long stator linear motors without a position encoder. After a discussion of the advantages and new possible applications with long stator linear motors, the fundamental voltage equations and the equation for the thrust force are shown. On the basis of this equation a current controller is presented which considers the characteristic of the flux during a transfer from the moveable unit through a segment. The essentials of an encoderless control approach are discussed and an expanded flux observer with an added voltage observer to detect the position is presented. In order to achieve a useful quality of the observed position during a transfer, a master-slave interchange strategy between the segments is presented. Finally the quality of the used position observer and the presented interchange strategy during a transfer from segment to segment is shown. The reached quality should be sufficient for many applications in the industrial environment.

\section{APPENDIX}

Motor-Segment: number of pole-pairs $=5$, rated voltage $=400 \mathrm{~V}$, rated current $=2.2 \mathrm{~A}$, rated speed $=0.96 \mathrm{~m} / \mathrm{s}$, rated force $=6.5 \mathrm{~N}$, pole pitch $=24 \mathrm{~mm}$, coil pitch $=40$ $\mathrm{mm}$, segment length $=240 \mathrm{~mm}$, stator resistance $=2.6$ Ohm per phase, stator inductance $=0.0125 \mathrm{H}$ per phase

\section{REFERENCES}

[1] P. Krause, O. Wasynczuk and S. Sudhoff, "Analysis of electric machinery and drive systems," IEEE Press Series on Power Engineering, 2002. http://dx.doi.org/10.1109/9780470544167

[2] Q. Ge, Y. Li and L. Kong, “A comparative study of FOC for long stator linear synchronous motor control, Electrical Machines and Systems". ICEMS. International Conference on, Volume, pp. 398$402,2007$.

[3] P. Vas, "Sensorless Vector and Direct Torque Control," Oxford Science Publications, 1998.

[4] S. Kim and S.-K. Sul, "Sensorless control of AC motor - Where are we now?," Electrical Machines and Systems (ICEMS), International Conference on, pp. 1-6, 2011. http://dx.doi.org/10.1109/icems.2011.6073316

[5] C. H. Ng, M. Rashed, P. Vas, F. Stronach, and P. MacConnell, "A novel mras current-based sensorless vector controlled pmlsm drive for low speed operation," in Electric Machines and Drives Conference, 2003. IEMDC'03. IEEE International, pp. 1889-1894 vol.3, June 2003.

[6] Y.-R. Chen, N. C. Cheung, and J. Wu, "Sensorless drive of permanent magnet linear motors using modified kalman filter," in Power Electronics Specialists Conference, 2001. PESC. 2001 IEEE 32nd Annual, vol. 4, pp.2009-2013 vol. 4, 2001.

[7] F. Cupertino, G. Pellegrino, P. Giangrande, and L. Salvatore, "Sensorless position control of permanent-magnet motors with pulsating current injection and compensation of motor end effects," IEEE Transactions on Industry Applications, vol. 47, no. 3, pp. 1371-1379, May 2011.

http://dx.doi.org/10.1109/TIA.2011.2126542

[8] W. Limei, G. Qingding, and Z. Jianfen, "Two-degree-of-freedom sensorless control of permanent magnet linear synchronous motor based on nonlinear observer," in Power Electronics and Motion Control Conference, 2004. IPEMC 2004. The 4th International, vol. 3, pp. 1427-1431 Vol.3, Aug 2004.

[9] K. Yoshida, Z. Dai, and M. Sato, "Sensorless dtc propulsion control of pm lsm vehicle," in Power Electronics and Motion Control Conference. Proceedings. IPEMC 2000. The Third International, vol. 1, 2000, pp. 191-196 vol.1, 2000.

http://dx.doi.org/10.1109/ipemc.2000.885355

[10]X. Wang and Q. Ge, "Speed sensorless control of a linear synchronous motor using state observer on d-q reference frame," in Electrical Machines and Systems, 2008. ICEMS 2008. International Conference on, pp. 1553-1557, Oct 2008.

[11]R. Leidhold and P. Mutschler, "Speed Sensorless Control of a Long-Stator Linear Synchronous Motor Arranged in Multiple Segments", Industrial Electronics, IEEE Transactions on, vol. 54, pp. 3246-3254, 2007.

http://dx.doi.org/10.1109/TIE.2007.905941

[12]R. Leidhold and P. Mutschler, "Sensorless position-control method based on magnetic saliencies for a Long-Stator Linear Synchronous-Motor", IEEE Industrial Electronics, IECON 2006 32nd Annual Conference on, pp. 781-786, 2006. http://dx.doi.org/10.1109/iecon.2006.347699

[13] S. Beineke, J. Schirmer, J. Lutz, H. Wertz, A. Bandhr and J. Kiel, J., "Implementation and applications of sensorless control for synchronous machines in industrial inverters", Sensorless Control for Electrical Drives (SLED), First Symposium on, pp. 64 -71, 2010.

[14]A. R. Weber and G. Steiner, "An accurate identification and compensation method for nonlinear inverter characteristics for AC motor drives", Instrumentation and Measurement Technology Conference (I2MTC), 2012 IEEE International, pp. 821-826, 2012. http://dx.doi.org/10.1109/i2mtc.2012.6229666 